\title{
All-Optical dc Nanotesla Magnetometry Using Silicon Vacancy Fine Structure in Isotopically Purified Silicon Carbide
}

\author{
D. Simin, ${ }^{1}$ V. A. Soltamov, ${ }^{2}$ A. V. Poshakinskiy, ${ }^{2}$ A. N. Anisimov, ${ }^{2}$ R. A. Babunts, ${ }^{2}$ \\ D. O. Tolmachev, ${ }^{2}$ E. N. Mokhov, ${ }^{2,5}$ M. Trupke, ${ }^{3}$ S. A. Tarasenko, ${ }^{2}$ A. Sperlich, ${ }^{1}$ \\ P. G. Baranov, ${ }^{2}$ V. Dyakonov, ${ }^{1,4, *}$ and G. V. Astakhov ${ }^{1, \dagger}$ \\ ${ }^{1}$ Experimental Physics VI, Julius-Maximilian University of Würzburg, 97074 Würzburg, Germany \\ ${ }^{2}$ Ioffe Physical-Technical Institute, 194021 St. Petersburg, Russia \\ ${ }^{3}$ Vienna Center for Quantum Science and Technology, Atominstitut, TU Wien, 1020 Wien, Austria \\ ${ }^{4}$ Bavarian Center for Applied Energy Research (ZAE Bayern), 97074 Würzburg, Germany \\ ${ }^{5}$ St. Petersburg National Research University of Information Technologies, \\ Mechanics and Optics, 197101 St. Petersburg, Russia
}

(Received 5 November 2015; revised manuscript received 27 March 2016; published 28 July 2016)

We uncover the fine structure of a silicon vacancy in isotopically purified silicon carbide $\left(4 \mathrm{H}^{28} \mathrm{SiC}\right)$ and reveal not yet considered terms in the spin Hamiltonian, originated from the trigonal pyramidal symmetry of this spin- $3 / 2$ color center. These terms give rise to additional spin transitions, which would be otherwise forbidden, and lead to a level anticrossing in an external magnetic field. We observe a sharp variation of the photoluminescence intensity in the vicinity of this level anticrossing, which can be used for a purely alloptical sensing of the magnetic field. We achieve dc magnetic field sensitivity better than $100 \mathrm{nT} / \sqrt{\mathrm{Hz}}$ within a volume of $3 \times 10^{-7} \mathrm{~mm}^{3}$ at room temperature and demonstrate that this contactless method is robust at high temperatures up to at least $500 \mathrm{~K}$. As our approach does not require application of radiofrequency fields, it is scalable to much larger volumes. For an optimized light-trapping waveguide of $3 \mathrm{~mm}^{3}$, the projection noise limit is below $100 \mathrm{fT} / \sqrt{\mathrm{Hz}}$.

DOI: 10.1103/PhysRevX.6.031014

\section{INTRODUCTION}

Vacancy-related color centers in the CMOS-compatible material silicon carbide $(\mathrm{SiC})$ are promising for chip-scale quantum technologies [1-9] based on ensembles [10-22] as well as on single centers [23-28]. Similar to the spin $S=1$ nitrogen-vacancy (NV) defect in diamond-which has become a standard solid-state system for the application of quantum sensing under ambient conditions [29-31] the silicon vacancy $\left(V_{\mathrm{Si}}\right)$ in $\mathrm{SiC}$ possesses selectively addressable spin states through optically detected magnetic resonance (ODMR) [4]. Unlike the spin-1 defects, the higher half-integer spin $S=3 / 2$ of $V_{\mathrm{Si}}[13,32]$ provides additional degree of freedom [33] and functionality [21], but these are usually unutilized. A major obstacle is that the structure of high-spin centers, being far more complicated, is not yet known. The level fine structure is the key to understanding spin dynamics and relaxation processes, which set limits for the performance of potential devices.

\footnotetext{
*dyakonov@physik.uni-wuerzburg.de

†astakhov@physik.uni-wuerzburg.de
}

Published by the American Physical Society under the terms of the Creative Commons Attribution 3.0 License. Further distribution of this work must maintain attribution to the author(s) and the published article's title, journal citation, and DOI.
Subject Areas: Condensed Matter Physics, Optoelectronics, Spintronics
Here, we reveal the fine structure of the $V_{\mathrm{Si}}$ ground and excited states (GS and ES, respectively) in external magnetic fields. We show that the $C_{3 v}$ point group of the $V_{\mathrm{Si}}$ defect gives rise to additional terms in the spin Hamiltonian, which have not been considered so far. Particularly, the trigonal pyramidal symmetry of the $V_{\mathrm{Si}}$ defect enables spin transitions with a change of the spin projection $\Delta m_{S}= \pm 2$. As compared to the commonly studied spin transitions with $\Delta m_{S}= \pm 1$, they are induced by counter circularly polarized radiation and their energies shift with twice the slope in a magnetic field. Moreover, we observe two GS level anticrossings (LAC) between the $m_{S}=-3 / 2$ and both $m_{S}=-1 / 2$ (GSLAC- 1$)$ and $m_{S}=$ $+1 / 2$ (GSLAC-2) spin sublevels. The GSLAC-2 can fundamentally occur for color centers with the spin $S \geq 3 / 2$ only. We develop a theory of the $V_{\mathrm{Si}}$ fine structure, which precisely takes into account the real atomic arrangement of the vacancy and quantitatively describes the experimental findings. The photoluminescence (PL) intensity demonstrates resonancelike behavior in the vicinity of LACs, and the sharpest resonance is detected for GSLAC2 , determined by the parameters related to the trigonal pyramidal symmetry of the $V_{\mathrm{Si}}$ center. In the following, we show that this optical phenomenon can be used to measure dc magnetic fields without the need to apply radiofrequency (rf) fields and we demonstrate sub-100-nT 
resolution within sub-1000 $\mu \mathrm{m}^{3}$. The effect is robust up to at least $500 \mathrm{~K}$, suggesting a simple, contactless method to monitor weak magnetic fields in a broad temperature range.

Our approach is easily scalable, and for a probe volume on the order of $1 \mathrm{~mm}^{3}$ with improved efficiency of optical pumping and light collection, we expect the magnetic field sensitivity to be about $100 \mathrm{fT}$ per square root of $\mathrm{Hz}$. While coming close to the sensitivity of other benchmark chipscale magnetic field sensors [34,35], this technique relies neither on rf fields, as for the NV defects in diamond [34], nor on vapor heating, as for the microfabricated rubidium cells [35]. Furthermore, the proposed method is not restricted to magnetic sensing and can potentially be extended for radio-frequency-free sensing of other physical quantities, particularly temperature and strain.

\section{EXPERIMENT}

In natural $\mathrm{SiC}$, the ODMR spectra of the $V_{\mathrm{Si}}$ defects are affected by the hyperfine interaction with the ${ }^{29} \mathrm{Si}$-isotope nuclear spin $I=1 / 2[13,36]$. In order to elude this interaction, we use isotopically purified $\mathrm{SiC}$ with above $99.0 \%$ of ${ }^{28} \mathrm{Si}$ nuclei with $I=0$. To obtain such a crystal, we first synthesize polycrystalline $\mathrm{SiC}$ with the use of silicon and carbon powders, the former being enriched with the ${ }^{28} \mathrm{Si}$ isotope. The polycrystalline substance is then used as a source for the growth of $4 \mathrm{H}^{2}{ }^{28} \mathrm{SiC}$ crystals by the sublimation method in a tantalum container [37]. The growth is performed in vacuum on $4 \mathrm{H}-\mathrm{SiC}$ substrates at a temperature of $2000^{\circ} \mathrm{C}$. The growth rate is approximately $0.25 \mathrm{~mm} / \mathrm{h}$. Afterwards, we polish out the substrate, obtaining the sample with a thickness of about $500 \mu \mathrm{m}$. In order to introduce the silicon vacancies, the sample is irradiated with neutrons in a nuclear reactor with a fluence of $1 \times 10^{16} \mathrm{~cm}^{-2}$, resulting in a nominal $V_{\mathrm{Si}}$ density of $2 \times 10^{14} \mathrm{~cm}^{-3}$ [27].

To optically address the $V_{\mathrm{Si}}$ spin states, we use a $785-\mathrm{nm}$ laser diode. The optical excitation followed by the spindependent recombination leads to a preferential population of the $m_{S}= \pm 1 / 2$ sublevels along the crystal symmetry $c$-axis [1]. The PL from $V_{\mathrm{Si}}$ occurs in the near-infrared spectral range [38], and it is selected and detected using a $\mathrm{Si}$ photodiode and a 900-nm long-pass filter. The PL intensity is spin dependent: in the case of the $V_{\mathrm{Si}}$ center we study here-the so-called $V 2$ center-it is higher when the system is in the $m_{S}= \pm 3 / 2$ states and lower when the system is in the $m_{S}= \pm 1 / 2$ states $[3,16,36]$. The laser beam is focused onto the sample using a $20 \times$ optical objective (N.A. $=0.3$ ), optimized for the near-infrared light, and the PL is collected through the same objective. The nominal excitation volume is $330 \mu \mathrm{m}^{3}$. To additionally manipulate the $V_{\mathrm{Si}}$ spin states, we apply a rf field, provided by a signal generator. The rf is then amplified, guided to a $500-\mu \mathrm{m}$-thick stripline and terminated with a $50-\Omega$ impedance. A static magnetic field can be applied in an arbitrary direction, using a 3D coil arrangement in combination with a permanent magnet. The field direction and strength are calibrated using a 3D Hall sensor.

In the absence of an external magnetic field, the $V_{\mathrm{Si}}$ GS is split in two Kramers degenerate spin sublevels $m_{S}=$ $\pm 3 / 2$ and $m_{S}= \pm 1 / 2$ with the zero-field splitting $2 D=$ $70 \mathrm{MHz}[16,36]$. When an external magnetic field $\mathbf{B}$ is applied parallel to the $c$-axis, the spin states are further split and the splitting is linear with $B_{z}(z \| c)$, as schematically shown in Fig. 1(a). A resonant rf field induces magnetic dipole transitions between the spin-split sublevels $( \pm 1 / 2 \rightarrow \pm 3 / 2)$, resulting in a change of the PL intensity $(\Delta \mathrm{PL})$. The room-temperature evolution of the ODMR spectrum (i.e., the rf-dependent ODMR contrast $\triangle \mathrm{PL} / \mathrm{PL}$ ) with the external magnetic field $B_{z}$ is presented in Fig. 1(b).

We first discuss the case of $B_{z}=0$ [Fig. 1(c)]. At a low rf power of $9 \mathrm{dBm}$, we detect a single ODMR line at a frequency $\nu_{0}=70 \mathrm{MHz}$, which is equal to $2 D$ in the GS. At much higher rf power $(40 \mathrm{dBm})$, we detect another ODMR line at a frequency of $410 \mathrm{MHz}$. Below, we establish that it corresponds to the zero-field splitting $2 D^{\prime}$ in the ES [Fig. 1(a)]. A similar resonance was observed previously [16] and ascribed to the Frenkel pair [39].

Upon application of a magnetic field $B_{z}$, one of the rfdriven transitions is $(-1 / 2 \rightarrow-3 / 2)$ with $\Delta m_{S}=-1$, and
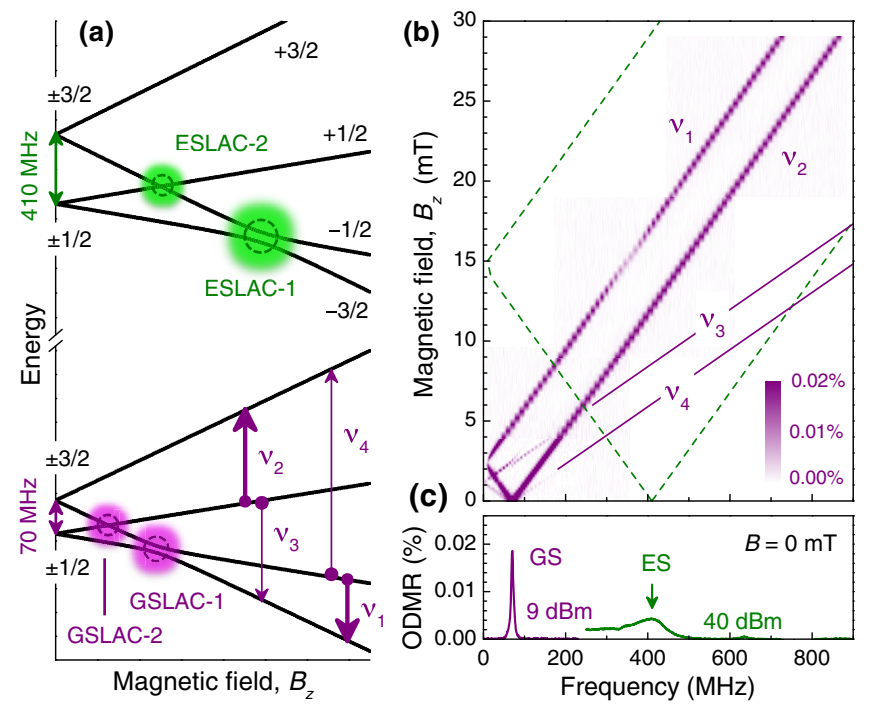

FIG. 1. (a) The GS $(2 D=70 \mathrm{MHz})$ and $\mathrm{ES}\left(2 D^{\prime}=410 \mathrm{MHz}\right)$ spin sublevels of the $V_{\mathrm{Si}}$ point defect in external magnetic field $B_{z} \| c$, assuming a weak perpendicular component $B_{\perp} \ll B_{z}$. The vertical arrows indicate rf-driven spin transitions, their thicknesses mirroring the contrasts of the corresponding ODMR lines. The magnetic field evolutions of the GS and ES are shown schematically, i.e., not to scale. (b) Magnetic field versus frequency evolution of the $V_{\mathrm{Si}}$ ODMR signal recorded at room temperature and at low rf power. The solid and dashed lines are the positions of the ODMR peaks calculated for the $\Delta m_{S}= \pm 2$ transitions in the GS and $\Delta m_{S}= \pm 1$ transitions in the ES, respectively. (c) Low-rf-power $(9 \mathrm{dBm})$ and high-rf-power (40 $\mathrm{dBm})$ ODMR spectra in zero magnetic field. 
the corresponding ODMR line $\nu_{1}=\left|\nu_{0}-g_{\|} \mu_{B} B_{z} / h\right|$ shifts linearly with $B_{z}$. Another rf-driven transition is $(+1 / 2 \rightarrow+3 / 2)$ with $\Delta m_{S}=+1$, and the corresponding ODMR line $\nu_{2}=\nu_{0}+g_{\|} \mu_{B} B_{z} / h$ shifts linearly towards higher frequencies. These two transitions are indicated by the thick arrows in Fig. 1(a) and the corresponding ODMR lines are clearly seen in Fig. 1(b), being in agreement with the previous results $[16,21]$. Remarkably, at a magnetic field $B_{G 1}=h \nu_{0} / g_{\|} \mu_{B}=2.5 \mathrm{mT}$, the frequency of the $\nu_{1}$ ODMR line [Fig. 1(b)] would tend to zero due to the level crossing in the ideal case. However, there is a gap opening at the crossing point caused by tiny perturbations of the transverse magnetic field component and/or nuclear field, resulting in GSLAC-1 [Fig. 1(a)].

We analyze the relative contrast of the $\nu_{1}$ and $\nu_{2}$ ODMR lines as a function of $B_{z}$ and observe two pronounced dips [Fig. 2(a)]. One of them is at $B_{G 1}=2.5 \mathrm{mT}$ (i.e., exactly at GSLAC-1) and the other one is at $B_{E 1}=15 \mathrm{mT}$. The dashed lines in Fig. 1(b) represent the calculated evolution of the ODMR spectrum associated with the $2 D^{\prime}=$ $410 \mathrm{MHz}$ resonance assuming the effective g-factor $g_{\|} \approx 2.0$. As expected, the ESLAC-1 occurs at $B_{E 1}$. We hence can reconstruct the ES spin structure, as shown in Fig. 1(a). It agrees with the conclusion drawn from another recent experiment [20]. The observation of the dip at $15 \mathrm{mT}$ in the $\nu_{1}$ rather than in the $\nu_{2}$ ODMR signal unambiguously

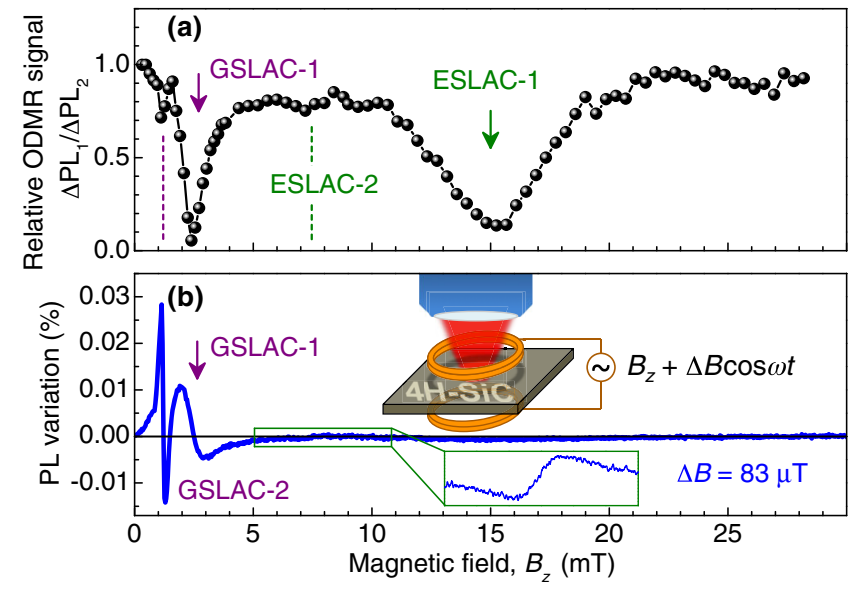

FIG. 2. (a) Relative strength of the $\nu_{1}$ and $\nu_{2}$ ODMR transitions $\left(\Delta \mathrm{PL}_{1} / \Delta \mathrm{PL}_{2}\right)$ as a function of the magnetic field $\mathbf{B}$ applied parallel to the $c$ axis of $4 \mathrm{H}-\mathrm{SiC}$. The arrows indicate the positions of GSLAC-1 (2.5 mT) and ESLAC-1 $(15 \mathrm{mT})$. The vertical dashed lines correspond to the expected positions of GSLAC-2 (lower field) and ESLAC-2 (higher field). (b) Lock-in detection of the PL variation $\triangle \mathrm{PL} / \mathrm{PL}$ as a function of the dc magnetic field $B_{z}$, where $\Delta \mathrm{PL}$ is caused by the application of an additional weak oscillating magnetic field $\Delta B$; i.e., $B_{z}+\Delta B \cos \omega t$ with $\Delta B=$ $83 \mu \mathrm{T}$ and $\omega / 2 \pi=5 \mathrm{kHz}$. The sharp resonancelike signal at $1.25 \mathrm{mT}$ corresponds to GSLAC-2. Resonant rf field is not applied. Upper inset: A scheme of the experiment. Lower inset: A detailed measurement in the magnetic field range corresponding to ESLAC-2. determines the order of the spin sublevels in the ES; i.e., the $m_{S}= \pm 3 / 2$ state has higher energy than the $m_{S}= \pm 1 / 2$ state $\left(D^{\prime}>0\right)$.

The appearance of dips in the ODMR signal of Fig. 2(a) is explained by modification of the optical pumping cycle in the vicinity of LACs either in the GS or ES, which, in turn, results in a change of the PL intensity, as previously reported for some other systems and techniques [40-45]. This suggests that LACs can be detected even without application of rf, simply by monitoring the PL intensity as a function of $B_{z}$. A schematic of this experiment is presented in the inset of Fig. 2(b). In order to increase the sensitivity, we modulate the dc magnetic field $B_{z}$ by additionally applying a small oscillating field $\Delta B \cos \omega t$ from the Helmholtz coils. The correspondingly oscillating PL signal detected by a photodiode is locked in, mirroring the first derivative of the PL on $B_{z}$. The experimental curve, recorded at a modulation frequency $\omega / 2 \pi=5 \mathrm{kHz}$ with a modulation depth $\Delta B=83 \mu \mathrm{T}$, is presented in Fig. 2(b). Surprisingly, in addition to the GSLAC-1, we detect a pronounced resonancelike behavior around $B_{G 2}=1.25 \mathrm{mT}$.

In order to understand the origin of two GSLACs, we measure the evolution of the ODMR spectrum as a function of $B_{z}$ with higher precision, in particular, with compensated transverse components of the geomagnetic field, $B_{x} \approx$ $B_{y} \approx 0 \pm \delta b \mu \mathrm{T}$. The compensation uncertainty depends on $B_{z}$ and is given by the alignment uncertainty $\delta \theta \approx 1^{\circ}$ as $\delta b=B_{z} \sin \delta \theta$. For instance, at the GSLAC-2, we have $\delta b=22 \mu \mathrm{T}$. The zoom-in of the spectral evolution in the vicinity of GSLAC-1 and GSLAC-2 is shown in Fig. 3(a). Beside the $\nu_{1}$ line, the ODMR spectrum contains an additional $\left(\nu_{3}\right)$ line, corresponding to the spin transition between the $m_{S}=+1 / 2$ and $m_{S}=-3 / 2$ GSs [sketched by a thin arrow in Fig. 1(a)]. The turning points of the $\nu_{1}$ and $\nu_{3}$ lines correspond to GSLAC-1 and GSLAC-2, respectively. The corresponding spectral shifts of the turning points $\nu_{1}^{\mathrm{AC}}$ and $\nu_{3}^{\mathrm{AC}}$, which are direct measures of the splitting values, are clearly detectable in Fig. 3(b) for various perpendicular components $B_{\perp}$ of the magnetic field. The level splittings at both GSLAC-1 and GSLAC-2 grow linearly with $B_{\perp}$, but with different slopes [Fig. 3(c)]. We emphasize that the amplitude of the $\nu_{3}$ line would rapidly tend to zero and the GSLAC-2 would disappear in an uniaxial model of the defect for magnetic fields being only slightly misaligned from the $c$ axis and, hence, should be vanishingly small in the experiment, assuming this model. Below, we present the exact calculation of the relative spin transition rates. Contrarily, the $\nu_{3}$ ODMR line is clearly detectable in Fig. 3(a) with the relative strength of the ODMR transition $\Delta \mathrm{PL}_{3} / \Delta \mathrm{PL}_{1}=$ $0.12 \pm 0.02$, and the most pronounced feature in Fig. 2(b) relates to the GSLAC-2.

While the magnetic disorder caused by hyperfine interaction with the residual ${ }^{29} \mathrm{Si}$ and ${ }^{13} \mathrm{C}$ nuclei or inaccurate orientation of the external magnetic field along the $c$-axis 

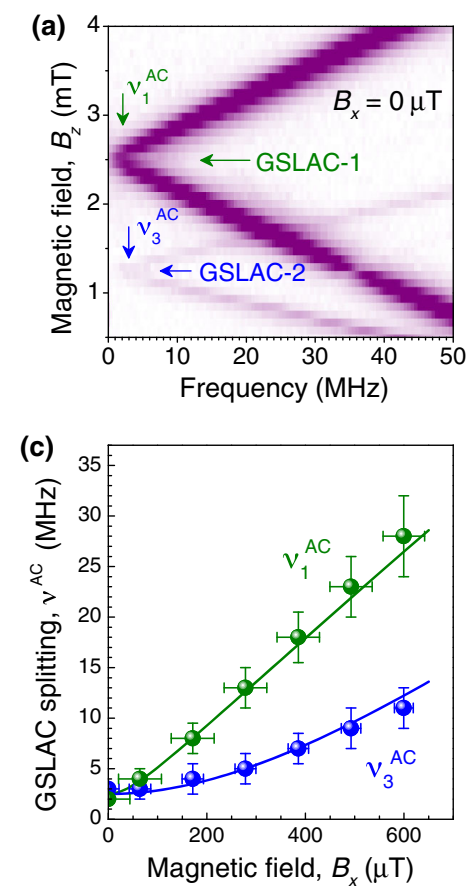

FIG. 3. (a) Evolution of the $\nu_{1}$ and $\nu_{3}$ ODMR lines in the vicinity of GSLACs as a function of the magnetic field $B_{z}$. The perpendicular components of the geomagnetic field are compensated. (b) Same as (a), but measured for nonzero $B_{x}$. The vertical arrows indicate the positions of the turning points $\nu_{1}^{\mathrm{AC}}$ and $\nu_{3}^{\mathrm{AC}}$, which are a direct measure of the level splittings at GSLAC-1 and GSLAC-2, respectively. (c) The GSLAC splittings as a function of $B_{x}$. The solid lines are calculations as explained in the text.

can, in principle, give rise to GSLAC-2 and the $\nu_{3}$ line, we conclude that they are not responsible for the above effects. Calculations (see Appendix A) yield that, for the average nuclear field seen by the $V_{\mathrm{Si}}$ centers of about $\left(h / 2 \mu_{B}\right) \times$ $1 \mathrm{MHz}[32,46]$ or magnetic field misalignment of $1^{\circ}$, the contrast ratio between the $\nu_{3}$ and $\nu_{1}$ ODMR lines is estimated to be $10^{-3}$, which is by 2 orders of magnitude lower than that observed in our experiments. Moreover, the amplitude of the $\nu_{3}$ line is the same in the natural (presented below) and isotopically purified $\mathrm{SiC}$ samples, while the abundance of the spin-carrying ${ }^{29} \mathrm{Si}$ nuclei differs by a factor of 5. Considering these results, a whole new approach to the spin structure of the $V_{\mathrm{Si}}$ defect is needed and is thoroughly assembled below.

\section{SILICON VACANCY FINE STRUCTURE}

Our findings can be explained in the framework of the spin Hamiltonian, which precisely takes into account the real microscopic $C_{3 v}$ group symmetry of the defect [47]. The effective Hamiltonian to the first order in the magnetic field can be presented as a sum of three contributions,

$$
\mathcal{H}=\mathcal{H}_{0}+\mathcal{H}_{1 \|}+\mathcal{H}_{1 \perp}
$$

where $\mathcal{H}_{0}$ is the Hamiltonian in zero magnetic field, and $\mathcal{H}_{1 \|} \propto B_{z}$ and $\mathcal{H}_{1 \perp} \propto \mathbf{B}_{\perp}=\left(B_{x}, B_{y}\right)$ are the magneticfield-induced terms. The Hamiltonians $\mathcal{H}_{0}, \mathcal{H}_{1 \|}$, and $\mathcal{H}_{1 \perp}$ can be constructed applying the theory of group representations [48]; see Appendix B for details. In the $C_{3 v}$ group, the magnetic field component $B_{z}$ and the spin operator $S_{z}$ transform under the irreducible representation $A_{2}$, the pairs of the in-plane components $\left(S_{x}, S_{y}\right)$ and $\left(B_{x}, B_{y}\right)$ transform under the representation $E$, and the Hamiltonian must be invariant (representation $A_{1}$ ). Using the multiplication table for the representations, one can construct all possible invariant combinations of the magnetic field components and the first, second, and third powers of the spin operator components. The fourth and higher powers of the spin-3/2 operator can be reduced to the operators of lower powers. Finally, taking into account that the Hamiltonian must be invariant with respect to the time reversal and, therefore, $\mathcal{H}_{0}$ is even in $\mathbf{S}$ while $\mathcal{H}_{1 \|}$ and $\mathcal{H}_{1 \perp}$ are odd in $\mathbf{S}$, we obtain

$$
\begin{aligned}
\mathcal{H}_{0}= & D\left(S_{z}^{2}-\frac{5}{4}\right), \\
\mathcal{H}_{1 \|}= & {\left[g_{\|} S_{z}+g_{2 \|} S_{z}\left(S_{z}^{2}-\frac{5}{4}\right)+g_{3 \|} \frac{S_{+}^{3}-S_{-}^{3}}{4 i}\right] \mu_{B} B_{z}, } \\
\mathcal{H}_{1 \perp}= & g_{\perp} \mu_{B} \mathbf{S}_{\perp} \cdot \mathbf{B}_{\perp}+2 g_{2 \perp} \mu_{B}\left\{\mathbf{S}_{\perp} \cdot \mathbf{B}_{\perp}, S_{z}^{2}-\frac{3}{4}\right\} \\
& +g_{3 \perp} \mu_{B} \frac{\left\{S_{+}^{2}, S_{z}\right\} B_{+}-\left\{S_{-}^{2}, S_{z}\right\} B_{-}}{2 i} .
\end{aligned}
$$

Here, $S_{x}, S_{y}, S_{z}$ are the spin-3/2 operators, $S_{\perp}=\left(S_{x}, S_{y}\right)$, $S_{ \pm}=S_{x} \pm i S_{y}, B_{ \pm}=B_{x} \pm i B_{y}, \quad\{A, B\}=(A B+B A) / 2$ is the symmetrized product, $z$ is parallel to the $c$ axis, $x$ and $y$ are the perpendicular axes with $y$ lying in a mirror reflection plane, and $\mu_{B}$ is the Bohr magneton. The six gfactors introduced in Eq. (2) are linearly independent in a structure of the $C_{3 v}$ point group. They can be determined from experimental data, as we do below, or obtained from $a b$ initio calculations, which is out of the scope of this paper. The difference $g_{\|}-g_{\perp}$ as well as the nonzero values of $D, g_{2 \|}$, and $g_{2 \perp}$ are due to nonequivalence of the $z$ axis and the perpendicular axes. The g-factors $g_{3 \|}$ and $g_{3 \perp}$ emerge due to the trigonal pyramidal symmetry of the defect. The Hamiltonian Eq. (2) can also be presented in the equivalent matrix form (see Appendix C).

The parameters of the Hamiltonian Eq. (2) can be determined from the experimental data. First, from the ODMR lines in the parallel $B_{z}$ [Fig. 1(b)] and perpendicular $B_{x}$ (Appendix D) magnetic fields, we confirm that to the second digit accuracy $g_{\|}, g_{\perp} \approx 2.0$, in agreement with earlier studies [36], and $\left|g_{2 \|}\right|,\left|g_{2 \perp}\right| \ll 1$. Then, using a procedure that is independent of the values of $g_{\|}$and $g_{\perp}$, we estimate the ratios $g_{2 \|} / g_{\|}$and $g_{2 \perp} / g_{\perp}$ using Eqs. (D3) and (D5) (see Appendix D). 
Furthermore, the Hamiltonian Eq. (2) describes the opening of spectral gaps due to the perpendicular field component $B_{\perp}$ at GSLAC-1 and GSLAC-2. The corresponding splittings $\Lambda_{1}$ and $\Lambda_{2}$ scale linearly with the perpendicular field for $\mu_{B} B_{\perp} \ll D$. Up to linear terms in $g_{2 \|}, g_{2 \perp}$ and quadratic terms in $g_{3 \|}, g_{3 \perp}$, the splittings in small fields are given by

$$
\begin{aligned}
& \Lambda_{1} \approx \sqrt{3}\left(1+\frac{g_{2 \perp}}{g_{\perp}}-\frac{g_{3 \|} g_{3 \perp}}{g_{\|} g_{\perp}}-\frac{g_{3 \|}^{2}}{8 g_{\|}^{2}}\right) g_{\perp} \mu_{B} B_{\perp}, \\
& \Lambda_{2} \approx \sqrt{3}\left(\frac{g_{3 \perp}}{g_{\perp}}+\frac{g_{3 \|}}{2 g_{\|}}\right) g_{\perp} \mu_{B} B_{\perp} .
\end{aligned}
$$

The GSLAC-2 emerges due to the trigonal asymmetry of the silicon vacancy, and the corresponding energy splitting is expected to be smaller than that in the GSLAC-1, $\Lambda_{2}<\Lambda_{1}$. Exactly such a behavior is observed in the experiment of Fig. 3. We fit the positions of the turning points as $h \nu_{1,3}^{\mathrm{AC}}=\left[\Lambda_{1,2}^{2}\left(B_{x}\right)+\Lambda_{0}^{2}\right]^{1 / 2}$, where $\Lambda_{0} / h=$ 2.5 MHz accounts for finite ODMR linewidth and inhomogeneity. From the best fit [the solid lines in Fig. 3(c)], we first obtain $g_{3 \perp}+g_{3 \|} / 2=0.5 \pm 0.2$ using the data for $\Lambda_{2}$ and then estimate the value $g_{3 \perp}-g_{3 \|} / 2=-0.1 \pm 0.4$ using the data for $\Lambda_{1}$. All the g-factors of the Hamiltonian Eq. (2) are summarized in Table I. It is instructive to compare the results with a high-symmetry defect of the $T_{d}$ point group, where one expects the relation $g_{3 \|} / g_{3 \perp}=2 / 3$ (see Appendix C).

We are now in a position to explain the appearance of the $\nu_{3}$ and $\nu_{4}$ ODMR lines in Figs. 1(b), 3(a), and 3(b), even when $B_{\perp}=0$. It follows from the Hamiltonian Eq. (2) that the matrix elements of the allowed magnetic dipole transitions have the form

$$
\begin{aligned}
& M_{\mp 3 / 2, \mp 1 / 2}=\frac{\sqrt{3}}{2}\left(1+\frac{g_{2 \perp}}{g_{\perp}}\right) g_{\perp} \mu_{B} B_{1, \sigma^{\mp}}, \\
& M_{\mp 3 / 2, \pm 1 / 2}=-i \frac{\sqrt{3}}{2}\left(\frac{g_{3 \perp}}{g_{\perp}}+\frac{g_{3 \|}}{2 g_{\|}}\right) g_{\perp} \mu_{B} B_{1, \sigma^{ \pm}},
\end{aligned}
$$

where $\mathbf{B}_{1}$ is the rf magnetic field and $B_{1, \sigma^{ \pm}}=B_{1, x} \mp i B_{1, y}$. The transitions $(+1 / 2 \rightarrow-3 / 2)$ and $(-1 / 2 \rightarrow+3 / 2)$, responsible for the $\nu_{3}$ and $\nu_{4}$ ODMR lines, respectively, occur due to the trigonal pyramidal symmetry of the spin$3 / 2$ defect and are induced by the $\sigma^{+}$and $\sigma^{-}$circularly

TABLE I. The g-factors in Hamiltonian Eq. (2) which, together with the zero-field splitting $2 D=70 \mathrm{MHz}$ and $g_{\|} \approx g_{\perp} \approx 2.0$, describe the GS fine structure of the silicon vacancy ( $V 2$ center in $4 \mathrm{H}-\mathrm{SiC}$ ) in a magnetic field.

\begin{tabular}{lccc}
\hline \hline$g_{2 \|}$ & $g_{2 \perp}$ & $g_{3 \perp}+g_{3 \|} / 2$ & $g_{3 \perp}-g_{3 \|} / 2$ \\
\hline $0.0 \pm 0.1$ & $0.0 \pm 0.1$ & $0.5 \pm 0.2$ & $-0.1 \pm 0.4$ \\
\hline \hline
\end{tabular}

polarized rf radiation. There are two microscopic contributions to these transitions: (i) coupling of the $m_{S}=+3 / 2$ and $m_{S}=-3 / 2$ states by the longitudinal static field $B_{z}$ (parameter $g_{3 \|}$ ) $[49,50]$ followed by the rf-driven transitions with $\Delta m= \pm 1$ and (ii) direct coupling of the $m_{S}=$ $+3 / 2$ and $m_{S}=-1 / 2$ as well as $m_{S}=-3 / 2$ and $m_{S}=$ $+1 / 2$ states by the transverse rf magnetic field (parameter $\left.g_{3 \perp}\right)$. Far from LACs, the ratio of the $\nu_{3}$ and $\nu_{1}$ ODMR line intensities for the linearly polarized $\mathrm{rf}$ field is given by $\left|M_{-3 / 2,1 / 2}\right|^{2} /\left|M_{-3 / 2,-1 / 2}\right|^{2} \approx\left(g_{3 \perp}+g_{3 \|} / 2\right)^{2} / 4$. Using $g_{3 \perp}+g_{3 \|} / 2=0.5$ from the fit of $\nu_{3}^{\mathrm{AC}}$ in Fig. 1(c), we obtain for the relative intensity 0.06 . It is somewhat smaller than the measured value of $\Delta \mathrm{PL}_{3} / \Delta \mathrm{PL}_{1} \approx 0.1$. A detailed comparison of the experimental and theoretical ODMR contrasts requires the study of the linewidths and resonance shapes in the vicinity of GSLACs, which is beyond the scope of the present paper. Given this uncertainty, we find the agreement between the theory and experiment satisfactory.

\section{ALL-OPTICAL MAGNETOMETRY}

Having established the fine structure, we propose to use its unique properties for all-optical magnetometry. The experimental procedure is straightforward and requires no rf field. First, we tune our system in the GSLAC-2, characterized by the narrowest resonance in Fig. 2(b). We then monitor the PL intensity through the lock-in inphase photovoltage $U_{X}$, which is simply proportional to the deviation of the measured magnetic field from the bias field $B_{G 2}$ (provided this deviation is small) [Fig. 4(a)]. By applying sub- $\mu \mathrm{T}$ magnetic fields, we calibrate the lock-in signal $U_{X} /\left(B_{z}-B_{G 2}\right)=39 \mu \mathrm{V} / \mu \mathrm{T}$ [Fig. 4(b)]. The quadrature component $U_{Y}$ of the lock-in signal, being
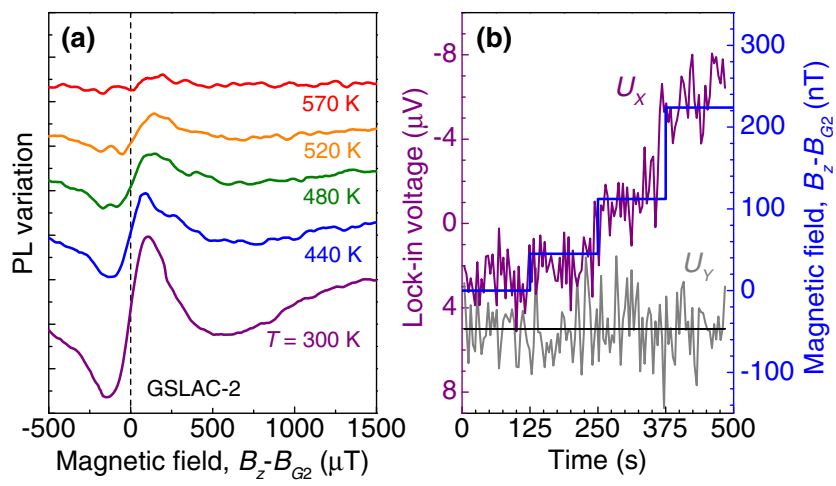

FIG. 4. (a) Lock-in detection of the PL variation in the vicinity of GSLAC-2 $\left(B_{G 2}=1.25 \mathrm{mT}\right)$ under application of a weak oscillating magnetic field, recorded at different temperatures. (b) The in-phase $U_{X}$ and quadrature $U_{Y}$ components of the lockin photovoltage (left axis) for different magnetic fields (right axis), increased in sub- $\mu \mathrm{T}$ steps every $125 \mathrm{~s}$. The horizontal line is the $U_{Y}$ mean value. The maximum field sensitivity is obtained for the modulation depth $\Delta B=200 \mu \mathrm{T}$ and modulation frequency $\omega / 2 \pi=511 \mathrm{~Hz}$. Temperature in (b) is $T=300 \mathrm{~K}$. 
independent of the magnetic field, is used to measure the noise level. Each data point in Fig. 4(b) corresponds to an integration time of $4 \mathrm{~s}$, and the dc magnetic field sensitivity is obtained to be $\delta B_{z}=87 \mathrm{nT} / \sqrt{\mathrm{Hz}}$. Indeed, magnetic fields below $100 \mathrm{nT}$ can be clearly resolved in Fig. 4(b).

We use an isotopically enriched crystal to exclude possible contributions related to the hyperfine interaction with ${ }^{29} \mathrm{Si}$ nuclei to the spin Hamiltonian of Eq. (2). Figure 5 demonstrates that the proposed all-optical magnetometry can also be performed using $\mathrm{SiC}$ with natural isotope abundance. By alignment of the bias magnetic field along the symmetry axis with an accuracy better that $1^{\circ}$ [inset of Fig. 5(a)], it is possible to clearly separate the spin-carrying isotope contributions. The GSLAC-2 is generally narrower and less sensitive to the magnetic field misalignment in comparison to the GSLAC-1, as can be seen in Fig. 5(b). It is indeed expected that $\Delta_{2}<\Delta_{1}$ because the peak-to-peak width $\Delta$, as determined in Fig. 5(a), scales with the LAC splitting $\Lambda$. By inserting the experimentally determined g-factors of Table I into Eq. (3), one obtains $\Lambda_{2}<\Lambda_{1}$.

The dynamic range of the proposed magnetometry is relatively small, several tens of $\mu \mathrm{T}$. It can be extended by applying a transverse magnetic field at the expense of sensitivity. On the other hand, there are many applications where large dynamic range is not required [51]. We would like to clarify that the proposed magnetometry is highly sensitive to one particular orientation of the magnetic field $\left(B_{z}\right)$ and, therefore, designed for applications where weak magnetic variations in a certain direction must be measured

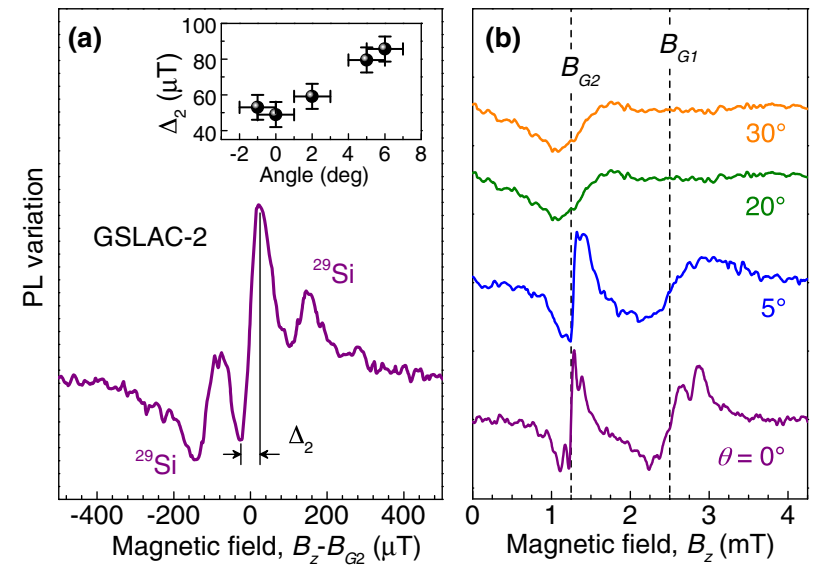

FIG. 5. (a) Lock-in detection of the PL variation in the vicinity of GSLAC-2 $\left(B_{G 2}=1.25 \mathrm{mT}\right)$, performed in $4 \mathrm{H}-\mathrm{SiC}$ with natural isotope abundance. Satellite resonances are due to the hyperfine interaction with ${ }^{29} \mathrm{Si}$ nuclei. Inset: Variation of the peak-to-peak width as a function of the magnetic field orientation. (b) PL variations at GSLAC-1 (at $B_{G 1}$ ) and GSLAC-2 (at $B_{G 2}$ ) for different magnetic field orientations with respect to the $c$-axis (polar angle $\theta$ ). $T=300 \mathrm{~K}$. The nonzero peak-to-peak width for $\theta=0^{\circ}$ is ascribed to magnetic fluctuations of the environment (nuclear spins and paramagnetic impurities) and magnetic field alignment uncertainty $\left(1^{\circ}\right)$. with high accuracy. The change of the sensitivity $\left(\propto \Delta_{2}\right)$ with the nonzero transverse field component $B_{\perp}$ can be found from the inset of Fig. 5(a). Using $\theta=$ $\arcsin \left(B_{\perp} / B_{G 2}\right)$, we empirically obtain for small angles $\delta B_{z}=\delta B_{z}^{(0)}\left(1+7.2 B_{\perp} / B_{G 2}\right)$. Here, $\delta B_{z}^{(0)}$ is the ideal sensitivity for a perfectly aligned magnetic field. For $B_{\perp}=$ $22 \mu \mathrm{T}\left(\theta \approx 1^{\circ}\right)$, the relative change of the sensitivity is $13 \%$. In order to align the magnetometer, it is necessary to conduct several preliminary measurements of magnetic field sweeps around $B_{G 2}$ in differently oriented bias magnetic fields, until the maximal slope is obtained. An advantage is that the LAC can be observed even for short spin lifetimes which occur, e.g., in excited states. The detection of the ODMR signal in those conditions may be difficult because it would require the application of highly intense rf fields. On the contrary, a variation of the PL intensity at ESLAC when the ODMR signal is not detectable has been clearly demonstrated using GaAs/AlGaAs superlattices [42]. Our approach is robust and can be applied in a broad temperature range up to $520 \mathrm{~K}$ [Fig. 4(a)]. A crucial factor for field sensitivity is the PL intensity and stability of the pump laser. The latter factor can be compensated using a balanced detection scheme. By increasing the irradiation fluence, the $V_{\mathrm{Si}}$ density can be increased by more than 2 orders of magnitude [27], and the projected sensitivity in this case is a few $\mathrm{nT} / \sqrt{\mathrm{Hz}}$ within the same volume of $330 \mu \mathrm{m}^{3}$. Alternatively, one can use light-trapping waveguides in bigger samples [34]. For a waveguide of $3 \mathrm{~mm} \times 3 \mathrm{~mm} \times 300 \mu \mathrm{m}$ with improved collection efficiency by 3 orders of magnitude [34] and a $V_{\mathrm{Si}}$ density of $4 \times 10^{16} \mathrm{~cm}^{-3}$ [27], we estimate the projection noise limit to be below $100 \mathrm{fT} / \sqrt{\mathrm{Hz}}$. In order to realize such an extremely high sensitivity, drift-compensation schemes [31,34] and magnetic noise screening similar to that usually used for optical magnetometry based on vapor cells [35] are necessary. The use of completely spin-free samples of high crystalline quality, containing ${ }^{28} \mathrm{Si}$ and ${ }^{12} \mathrm{C}$ isotopes only, can lead to further improvement due to the suppression of magnetic fluctuations caused by nuclear spins. In addition, rhombic $\mathrm{SiC}$ polytypes (15R) with even lower magnetic fields corresponding to the GSLACs [46] may have advantages compared to hexagonal $4 \mathrm{H}-\mathrm{SiC}$.

In conclusion, we reconstruct for the first time the fine structure of the $V_{\mathrm{Si}}$ center in $4 \mathrm{H}-\mathrm{SiC}$, quantifying its contributions. The presence and field-dependent behavior of two GSLACs can now be theoretically described and predicted. Our results on the spin Hamiltonian as well as the approach to study the fine structures of localized states are general. They can be directly applied for other spin-3/2 systems with the trigonal pyramidal symmetry known in the solid state, such as other color centers in zinc-blendetype crystals or $\Gamma_{8}$-band holes in quantum dots [50]. They can also be straightforwardly generalized to high-spin 
states such as transition-metal impurities in semiconductor structures of low spatial symmetry.

These findings are directly translated to a working application, namely, an all-optical magnetometry with sub-100-nT sensitivity. This is a general concept of alloptical sensing without rf fields, as it can be used to measure various physical quantities, such as temperature and strain, through their effect on the zero-field splitting and hence on the magnetic fields corresponding to LACs. An intriguing possibility is to image the PL from a SiC wafer onto a CCD camera to visualize magnetic fields with temporal and spatial resolution. Our results may potentially be applied for biomedical imaging and geophysical surveying, especially when rf fields cannot be applied.

\section{ACKNOWLEDGMENTS}

This work has been supported by the German Research Foundation (DFG) under Grant No. AS 310/4, by the BMBF under the ERA.Net RUS Plus project "DIABASE," and by the RFBR No. 14-02-91344; V. A. S., A. N. A., R. A. B., and P. G. B. are grateful for the financial support of the Russian Science Foundation No. 14-12-00859; A. V. P. and S. A. T. are grateful for the support of RFBR No. 14-0200168, RF president grant SP-2912.2016.5, and "Dynasty" Foundation. This publication was supported by the Open Access Publication Fund of the University of Würzburg.

\section{APPENDIX A: SPIN TRANSITIONS IN THE UNIAXIAL MODEL}

In the uniaxial approximation, the effective Hamiltonian of a spin center has the form

$$
H=D\left(S_{z}^{2}-\frac{5}{4}\right)+g_{\|} \mu_{B} S_{z} B_{z}+g_{\perp} \mu_{B} \mathbf{S}_{\perp} \cdot \mathbf{B}_{\perp},
$$

where $g_{\|}$and $g_{\perp}$ are the longitudinal and transverse g-factors, $B_{z}$ and $\mathbf{B}_{\perp}=\left(B_{x}, B_{y}\right)$ are the longitudinal and transverse components of the magnetic field, respectively, and $\mathbf{S}$ is the vector composed of the spin-3/2 operators $S_{x}, S_{y}$, and $S_{z}$.

The transverse component of the magnetic field caused by inaccurate orientation of the external magnetic field along the $c$ axis and/or originated from hyperfine interaction with nuclei couples the $+1 / 2$ and $-1 / 2$ spin states as well as the $-1 / 2$ and $-3 / 2$ spin states, thereby allowing the $\nu_{3}$ ODMR line. Straightforward perturbation-theory calculations show that the ratio between the intensities of the $\nu_{3}$ and $\nu_{1}$ ODMR lines determined by the corresponding matrix elements of the transitions has the form

$$
\frac{\left|M_{-3 / 2,+1 / 2}\right|^{2}}{\left|M_{-3 / 2,-1 / 2}\right|^{2}}=\left(\frac{2 D}{2 D-g_{\|} \mu_{B} B_{z}} \frac{g_{\perp} B_{\perp}}{g_{\|} B_{z}}\right)^{2} .
$$

The experimental value of the relative OMDR contrast is about 0.1 . To obtain such a contrast, e.g., for $g_{\|} \mu_{B} B_{z}=D$, one has to assume that $B_{\perp} / B_{z} \approx 0.16$, which corresponds to an angle of approximately $10^{\circ}$ between the total magnetic field acting upon spin centers and the $c$ axis or $B_{\perp} \approx 0.2 \mathrm{mT}$ for $B_{z}=1.25 \mathrm{mT}$. Such a transverse magnetic field is an order of magnitude larger than the average nuclear field seen by the $V_{\mathrm{Si}}$ centers [46]. The precision of the external magnetic orientation in our experiments is also by an order of magnitude better [inset of Fig. 5(a)]. For such a precision, the contrast ratio between the $\nu_{3}$ and $\nu_{1}$ ODMR lines is estimated from Eq. (A2) to be at most 0.001 , which is by 2 orders of magnitude lower than the experimentally determined ratio.

\section{APPENDIX B: EFFECTIVE HAMILTONIAN FOR THE SPIN-3/2 CENTER OF THE $C_{3 v}$ POINT GROUP}

We construct the effective spin Hamiltonian using the theory of group representations [48]. In the $C_{3 v}$ point group, there are three irreducible representations commonly denoted as $A_{1}, A_{2}$, and $E$. Accordingly, all physical quantities can be decomposed into the irreducible representations in accordance with their symmetry properties. The magnetic field components $B_{\alpha}(\alpha=x, y, z)$ and all possible linearly independent combinations of the spin operator components $S_{\alpha}$ are decomposed into the irreducible representations as follows:

$$
\begin{aligned}
A_{1}: & S_{z}^{2} ; S_{x}\left(3 S_{y}^{2}-S_{x}^{2}\right), \\
A_{2}: & B_{z} ; S_{z} ; S_{z}^{3} ; S_{y}\left(3 S_{x}^{2}-S_{y}\right), \\
E: & \left(B_{x}, B_{y}\right) ;\left(S_{x}, S_{y}\right) ;\left(S_{y} S_{z},-S_{x} S_{z}\right) ;\left(S_{x} S_{z}^{2}, S_{y} S_{z}^{2}\right) \\
& \left(S_{x}^{2}-S_{y}^{2},-2\left\{S_{x}, S_{y}\right\}\right) ;\left(2\left\{S_{x}, S_{y}\right\} S_{z},\left(S_{x}^{2}-S_{y}^{2}\right) S_{z}\right) .
\end{aligned}
$$

All other combinations of the spin operator components can be expressed via the above ones, taking into account the identity $S_{x}^{2}+S_{y}^{2}+S_{z}^{2}=15 / 4$ and the fact that the fourth and higher powers of the spin-3/2 operator components can be reduced to the operators of lower powers.

The effective Zeeman Hamiltonian is constructed as the sum of all possible products of the magnetic field components and the spin operator combinations that are invariant with respect to (i) symmetry operations of the point group and (ii) time reversal. Each invariant contribution is multiplied by a prefactor that has a physical sense of an effective g-factor component. The condition (i) implies that the invariant products are constructed from quantities belonging to the same irreducible representation. The condition (ii) implies that the Zeeman Hamiltonian is odd in $\mathbf{S}$. Taking both conditions into account, we obtain six linearly independent contributions to the Zeeman Hamiltonian, which are given in Eq. (2).

A similar procedure based on the theory of group representations can be applied to construct the 
Hamiltonian describing the effect of strain on the $V_{\mathrm{Si}}$ spin center, hyperfine interaction, etc. The trigonal pyramidal symmetry of the defect gives rise to additional terms in the spin Hamiltonian, which are absent in the uniaxial model.

$$
\mathcal{H}=\left(\begin{array}{cc}
D+\frac{3}{2}\left(1+\frac{g_{2 \|}}{g_{\|}}\right) g_{\|} \mu_{B} B_{z} & \frac{\sqrt{3}}{2}\left(1+\frac{g_{2 \perp}}{g_{\perp}}\right) g_{\perp} \mu_{B} B_{-} \\
\frac{\sqrt{3}}{2}\left(1+\frac{g_{2 \perp}}{g_{\perp}}\right) g_{\perp} \mu_{B} B_{+} & -D+\frac{1}{2}\left(1-\frac{g_{2 \|}}{g_{\|}}\right) g_{\|} \mu_{B} B_{z} \\
i \frac{\sqrt{3}}{2} g_{3 \perp} \mu_{B} B_{-} & \left(1-\frac{g_{2 \perp}}{g_{\perp}}\right) g_{\perp} \mu_{B} B_{+} \\
i \frac{3}{2} g_{3 \|} \mu_{B} B_{z} & -i \frac{\sqrt{3}}{2} g_{3 \perp} \mu_{B} B_{-}
\end{array}\right.
$$

where $B_{ \pm}=B_{x} \pm i B_{y}=B_{\perp} e^{ \pm i \phi}$, with $\phi$ denoting the azimuthal angle of $\mathbf{B}$. To derive this matrix, we use the explicit form of the spin-3/2 matrices:

$$
\begin{aligned}
& S_{x}=\left(\begin{array}{cccc}
0 & \frac{\sqrt{3}}{2} & 0 & 0 \\
\frac{\sqrt{3}}{2} & 0 & 1 & 0 \\
0 & 1 & 0 & \frac{\sqrt{3}}{2} \\
0 & 0 & \frac{\sqrt{3}}{2} & 0
\end{array}\right), \\
& S_{y}=\left(\begin{array}{cccc}
0 & -i \frac{\sqrt{3}}{2} & 0 & 0 \\
i \frac{\sqrt{3}}{2} & 0 & -i & 0 \\
0 & i & 0 & -i \frac{\sqrt{3}}{2} \\
0 & 0 & i \frac{\sqrt{3}}{2} & 0
\end{array}\right), \\
& S_{z}=\left(\begin{array}{ccccc}
\frac{3}{2} & 0 & 0 & 0 \\
0 & \frac{1}{2} & 0 & 0 \\
0 & 0 & -\frac{1}{2} & 0 \\
0 & 0 & 0 & -\frac{3}{2}
\end{array}\right) .
\end{aligned}
$$

\section{Zeeman splitting of spin sublevels}

Application of a magnetic field along the $c$-axis leads to the splitting of the spin sublevels. The energies of the states with the spin projections $m_{S}= \pm 1 / 2$ and $m_{S}= \pm 3 / 2$ are given by

\section{APPENDIX C: FINE STRUCTURE OF SPIN-3/2 CENTERS OF THE $C_{3 v}$ POINT GROUP}

The spin Hamiltonian given by Eqs. (1) and (2) can be rewritten in the equivalent matrix form:

$$
\left.\begin{array}{cc}
-i \frac{\sqrt{3}}{2} g_{3 \perp} \mu_{B} B_{+} & -i \frac{3}{2} g_{3 \|} \mu_{B} B_{z} \\
\left(1-\frac{g_{2 \perp}}{g_{\perp}}\right) g_{\perp} \mu_{B} B_{-} & i \frac{\sqrt{3}}{2} g_{3 \perp} \mu_{B} B_{+} \\
-D-\frac{1}{2}\left(1-\frac{g_{2 \|}}{g_{\|}}\right) g_{\|} \mu_{B} B_{z} & \frac{\sqrt{3}}{2}\left(1+\frac{g_{2 \perp}}{g_{\perp}}\right) g_{\perp} \mu_{B} B_{-} \\
\frac{\sqrt{3}}{2}\left(1+\frac{g_{2 \perp}}{g_{\perp}}\right) g_{\perp} \mu_{B} B_{+} & D-\frac{3}{2}\left(1+\frac{g_{2 \|}}{g_{\|}}\right) g_{\|} \mu_{B} B_{z}
\end{array}\right),
$$

$$
\begin{aligned}
& E_{ \pm 1 / 2}=-D \pm \frac{1}{2} g_{\|, 1 / 2} \mu_{B} B_{z}, \\
& E_{ \pm 3 / 2}=D \pm \frac{3}{2} g_{\|, 3 / 2} \mu_{B} B_{z},
\end{aligned}
$$

where the effective g-factors are

$$
\begin{aligned}
& g_{\|, 1 / 2}=g_{\|}-g_{2 \|}, \\
& g_{\|, 3 / 2}=\sqrt{\left(g_{\|}+g_{2 \|}\right)^{2}+g_{3 \|}^{2}} .
\end{aligned}
$$

The spin sublevel $m_{S}=-3 / 2$ crosses the spin sublevels $m_{S}=-1 / 2$ and $m_{S}=+1 / 2$ at the magnetic fields

$$
B_{G 1}=\frac{4 D}{\left(3 g_{\|, 3 / 2}-g_{\|, 1 / 2}\right) \mu_{B}}
$$

$$
\text { and } \quad B_{G 2}=\frac{4 D}{\left(3 g_{\|, 3 / 2}+g_{\|, 1 / 2}\right) \mu_{B}}
$$

respectively.

As we describe in the main text, application of a small additional perpendicular magnetic field $B_{\perp}$ leads to level anticrossings, GSLAC-1 and GSLAC-2 [Fig. 1(a)], at $B_{G 1}$ and $B_{G 2}$, respectively.

In a magnetic field applied perpendicular to the $c$-axis, $B_{\perp}=\left(B_{x}^{2}+B_{y}^{2}\right)^{1 / 2}$ and $B_{z}=0$, the energy spectrum is given by

$$
\begin{aligned}
& E_{3 / 2}= \pm \frac{1}{2}\left(g_{\perp}-g_{2 \perp}\right) \mu_{B} B_{\perp}+\frac{1}{2} \sqrt{\left[2 D \mp\left(g_{\perp}-g_{2 \perp}\right) \mu_{B} B_{\perp}\right]^{2}+3\left[\left(g_{\perp}+g_{2 \perp}\right)^{2}+g_{3 \perp}^{2}\right] \mu_{B}^{2} B_{\perp}^{2}}, \\
& E_{1 / 2}= \pm \frac{1}{2}\left(g_{\perp}-g_{2 \perp}\right) \mu_{B} B_{\perp}-\frac{1}{2} \sqrt{\left[2 D \mp\left(g_{\perp}-g_{2 \perp}\right) \mu_{B} B_{\perp}\right]^{2}+3\left[\left(g_{\perp}+g_{2 \perp}\right)^{2}+g_{3 \perp}^{2}\right] \mu_{B}^{2} B_{\perp}^{2}}
\end{aligned}
$$


Particularly, for small magnetic fields $\left(\mu_{B} B_{\perp} \ll 2 D\right)$, the linear-in- $B_{\perp}$ splitting is described by the effective g-factors:

$$
\begin{aligned}
& g_{\perp, 3 / 2}=0, \\
& g_{\perp, 1 / 2}=2\left(g_{\perp}-g_{2 \perp}\right) .
\end{aligned}
$$

\section{Relation between g-factors in the $T_{d}$ point group}

The spatial arrangement of carbon atoms around the single silicon vacancy is close to the tetragonal structure, which is described by the $T_{d}$ point group symmetry. The $T_{d}$ group symmetry is higher than the real $C_{3 v}$ group symmetry of the vacancy but properly takes into account the threefold rotation $c$ axis and allows for nonzero values of both $g_{3 \|}$ and $g_{3 \perp}$. Thus, one can expect that the relation between $g_{3 \|}$ and $g_{3 \perp}$ of the Si vacancy is close to that for the defect of the $T_{d}$ point group.

In the $T_{d}$ point group, the effective Zeeman Hamiltonian of the spin-3/2 defect in the cubic axes $x^{\prime}\left\|[100], y^{\prime}\right\|[010]$, and $z^{\prime} \|[001]$ is described by two linearly independent parameters $g$ and $q$ and reads [52]

$$
\begin{aligned}
\mathcal{H}_{T_{d}}= & g \mu_{B} \mathbf{S} \cdot \mathbf{B} \\
& +q \mu_{B}\left(J_{x^{\prime}}^{3} B_{x^{\prime}}+J_{y^{\prime}}^{3} B_{y^{\prime}}+J_{z^{\prime}}^{3} B_{z^{\prime}}-\frac{41}{20} \mathbf{S} \cdot \mathbf{B}\right) .
\end{aligned}
$$

In order to obtain the Hamiltonian in the axes $x \|[1 \overline{1} 0]$, $y \|[11 \overline{2}]$, and $z \|[111]$, relevant to the vacancy orientation, we use the relation between the components of the vector $\mathbf{B}$ in two coordinate frames,

$$
\begin{aligned}
& B_{x^{\prime}}=\frac{1}{\sqrt{2}} B_{x}+\frac{1}{\sqrt{6}} B_{y}+\frac{1}{\sqrt{3}} B_{z}, \\
& B_{y^{\prime}}=-\frac{1}{\sqrt{2}} B_{x}+\frac{1}{\sqrt{6}} B_{y}+\frac{1}{\sqrt{3}} B_{z}, \\
& B_{z^{\prime}}=-\frac{2}{\sqrt{6}} B_{y}+\frac{1}{\sqrt{3}} B_{z}
\end{aligned}
$$

and similar equations for the components of the spin operator $\mathbf{S}$. This yields

$$
\mathcal{H}_{T_{d}}=\mu_{B}\left(\begin{array}{cccc}
\left(\frac{3}{2} g-\frac{1}{5} q\right) B_{z} & \frac{\sqrt{3}}{2}\left(g+\frac{1}{5} q\right) B_{-} & -\sqrt{\frac{3}{8}} i q B_{+} & -\frac{1}{\sqrt{2}} i q B_{z} \\
\frac{\sqrt{3}}{2}\left(g+\frac{1}{5} q\right) B_{+} & \left(\frac{1}{2} g+\frac{3}{5} q\right) B_{z} & \left(g-\frac{3}{10} q\right) B_{-} & \sqrt{\frac{3}{8}} i q B_{+} \\
\sqrt{\frac{3}{8}} i q B_{-} & \left(g-\frac{3}{10} q\right) B_{+} & -\left(\frac{1}{2} g+\frac{3}{5} q\right) B_{z} & \frac{\sqrt{3}}{2}\left(g+\frac{1}{5} q\right) B_{-} \\
\frac{1}{\sqrt{2}} i q B_{z} & -\sqrt{\frac{3}{8}} i q B_{-} & \frac{\sqrt{3}}{2}\left(g+\frac{1}{5} q\right) B_{+} & -\left(\frac{3}{2} g-\frac{1}{5} q\right) B_{z}
\end{array}\right)
$$

Comparing the Hamiltonians Eqs. (C1) and (C16), we obtain that $g_{3 \|}$ and $g_{3 \perp}$ are related to each other by

$$
g_{3 \|} / g_{3 \perp}=2 / 3
$$

for a defect of the $T_{d}$ group symmetry.

\section{APPENDIX D: EXTRACTING THE FINE STRUCTURE PARAMETERS}

To obtain the value of $g_{2 \|}$, we use the ODMR spectra, recorded in the magnetic field applied parallel to the $c$-axis. The linear shift of the ODMR lines is given by

$$
\begin{aligned}
\nu_{1,2} & =\nu_{0} \pm\left(\frac{3}{2} g_{\|, 3 / 2}-\frac{1}{2} g_{\|, 1 / 2}\right) \mu_{B} B_{z} / h \\
& \approx \nu_{0} \pm\left(g_{\|}+2 g_{2 \|}+3 g_{3 \|}^{2} / 4 g_{\|}\right) \mu_{B} B_{z} / h
\end{aligned}
$$

$$
\begin{aligned}
\nu_{3,4} & =\nu_{0} \pm\left(\frac{3}{2} g_{\|, 3 / 2}+\frac{1}{2} g_{\|, 1 / 2}\right) \mu_{B} B_{z} / h \\
& \approx \nu_{0} \pm\left(2 g_{\|}+g_{2 \|}+3 g_{3 \|}^{2} / 4 g_{\|}\right) \mu_{B} B_{z} / h,
\end{aligned}
$$

where $\nu_{0}=2 D / h$. The experimentally measured ratio of the magnetic fields corresponding to the GSLAC-2 and GSLAC-1 points, $B_{G 2} / B_{G 1}=0.503 \pm 0.005$, independent of the magnetic field calibration, allows us to extract the value of $g_{2 \|} / g_{\|}$using the formula

$$
\frac{B_{G 2}}{B_{G 1}} \approx \frac{1}{2}+\frac{3 g_{2 \|}}{4 g_{\|}}+\frac{3 g_{3 \|}^{2}}{16 g_{\|}^{2}} .
$$

For the first iteration we neglect the term $\propto g_{3 \|}^{2}$.

To determine $g_{2 \perp}$, we exploit the evolution of the ODMR spectrum in the magnetic field $B_{\perp}$ applied perpendicular to the $c$ axis, presented in Fig. 6(a). From Eq. (C11), we obtain the positions of the $\nu_{1}$ and $\nu_{2}$ ODMR lines up to $B_{\perp}^{2}$ : 


$$
\begin{aligned}
\nu_{1,2}= & \nu_{0} \mp\left(g_{\perp}-g_{2 \perp}\right) \frac{\mu_{B} B_{\perp}}{h} \\
& +\frac{3\left[\left(g_{\perp}+g_{2 \perp}\right)^{2}+g_{3 \perp}^{2}\right]}{2 \nu_{0}}\left(\frac{\mu_{B} B_{\perp}}{h}\right)^{2} .
\end{aligned}
$$

One can represent the quadratic shift $\left(\nu_{2}+\nu_{1}\right) / 2$ versus the Zeeman splitting $\nu_{2}-\nu_{1}=2\left(g_{\perp}-g_{2 \perp}\right) B_{\perp} / h$, using the theoretical expression

$$
\begin{aligned}
\frac{\nu_{2}+\nu_{1}}{2}-\nu_{0} & =\frac{3\left[\left(g_{\perp}+g_{2 \perp}\right)^{2}+g_{3 \perp}^{2}\right]}{8\left(g_{\perp}-g_{2 \perp}\right)^{2}} \frac{\left(\nu_{2}-\nu_{1}\right)^{2}}{\nu_{0}} \\
& \approx\left(1+\frac{4 g_{2 \perp}}{g_{\perp}}+\frac{g_{3 \perp}^{2}}{g_{\perp}^{2}}\right) \frac{3\left(\nu_{2}-\nu_{1}\right)^{2}}{8 \nu_{0}} .
\end{aligned}
$$

From the linear fit of the experimental data in Fig. 6(b), we obtain the value of $g_{2 \perp} / g_{\perp}$. Again, for the first iteration we neglect the term $\propto g_{3 \perp}^{2}$.

Finally, we use the GSLAC-1 and GSLAC-2 splittings, described by Eq. (3) to determine the values of $g_{3 \|}$ and $g_{3 \perp}$. By iterating the described procedure, we obtain the values of the g-factors, as presented in Table I.
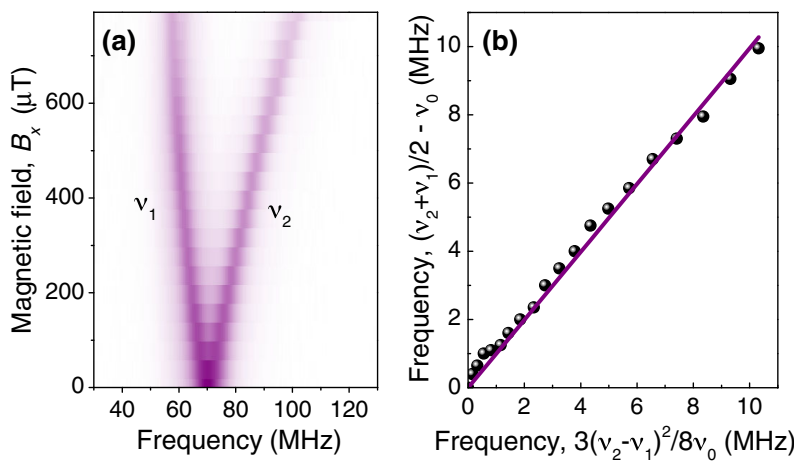

FIG. 6. (a) Evolution of the ODMR spectrum as a function of the perpendicular magnetic field $B_{\perp}=B_{x}\left(B_{y}=0\right)$. (b) Points represent the quadratic shift $\left(\nu_{2}+\nu_{1}\right) / 2-\nu_{0}$ versus the Zeeman splitting in terms of $3\left(\nu_{2}-\nu_{1}\right)^{2} / 8 \nu_{0}$. Solid line is a linear fit to Eq. (D5) with a slope of $0.995 \pm 0.01$.

\section{APPENDIX E: ELECTRON SPIN RESONANCE}

The spin transition rates induced by the rf magnetic field $\mathbf{B}_{1}$ are determined by the matrix elements of Hamiltonian Eq. (C1). For a static magnetic field $\mathbf{B} \| z$, the matrix elements of the transitions up to linear in $g_{2 \|}, g_{2 \perp}, g_{3 \|}$, and $g_{3 \perp}$ terms are given by Eqs. (4) and (5) in the main text, and the full expressions have the form

$$
\begin{aligned}
M_{-3 / 2,-1 / 2} & =\sqrt{\frac{3}{8}}\left[\left(g_{\perp}+g_{2 \perp}\right) \sqrt{1+\frac{g_{\|}+g_{2 \|}}{g_{\|, 3 / 2}}}+\frac{g_{3 \|} g_{3 \perp}}{\sqrt{g_{\|, 3 / 2}} \sqrt{g_{\|}+g_{2 \|}+g_{\|, 3 / 2}}}\right] \mu_{B}\left(B_{1, x}+i B_{1, y}\right), \\
M_{3 / 2,1 / 2} & =\sqrt{\frac{3}{8}}\left[\left(g_{\perp}+g_{2 \perp}\right) \sqrt{1+\frac{g_{\|}+g_{2 \|}}{g_{\|, 3 / 2}}}+\frac{g_{3 \|} g_{3 \perp}}{\sqrt{g_{\|, 3 / 2}} \sqrt{g_{\|}+g_{2 \|}+g_{\|, 3 / 2}}}\right] \mu_{B}\left(B_{1, x}-i B_{1, y}\right), \\
M_{-3 / 2,1 / 2} & =-i \sqrt{\frac{3}{8}}\left[\frac{g_{3 \|}\left(g_{\perp}+g_{2 \perp}\right)}{\sqrt{g_{\|, 3 / 2}} \sqrt{g_{\|}+g_{2 \|}+g_{\|, 3 / 2}}}+g_{3 \perp} \sqrt{1+\frac{g_{\|}+g_{2 \|}}{g_{\|, 3 / 2}}}\right] \mu_{B}\left(B_{1, x}-i B_{1, y}\right), \\
M_{3 / 2,-1 / 2} & =-i \sqrt{\frac{3}{8}}\left[\frac{g_{3 \|}\left(g_{\perp}+g_{2 \perp}\right)}{\sqrt{g_{\|, 3 / 2}} \sqrt{g_{\|}+g_{2 \|}+g_{\|, 3 / 2}}}+g_{3 \perp} \sqrt{1+\frac{g_{\|}+g_{2 \|}}{g_{\|, 3 / 2}}}\right] \mu_{B}\left(B_{1, x}+i B_{1, y}\right) .
\end{aligned}
$$

The matrix element for $(-1 / 2 \leftrightarrow+1 / 2)$ has the form $M_{1 / 2,-1 / 2}=\left(g_{\perp}-g_{2 \perp}\right) \mu_{B}\left(B_{1, x}-i B_{1, y}\right)$, while the spin transition $(-3 / 2 \leftrightarrow+3 / 2)$ is forbidden for $\mathbf{B} \| z$.

[1] P. G. Baranov, A. P. Bundakova, I. V. Borovykh, S. B. Orlinskii, R. Zondervan, and J. Schmidt, Spin Polarization Induced by Optical and Microwave Resonance Radiation in a Si Vacancy in SiC: A Promising Subject for the Spectroscopy of Single Defects, J. Exp. Theor. Phys. Lett. 86, 202 (2007).
[2] J. R. Weber, W. F. Koehl, J. B. Varley, A. Janotti, B. B. Buckley, C. G. Van de Walle, and D. D. Awschalom, Quantum Computing with Defects, Proc. Natl. Acad. Sci. U.S.A. 107, 8513 (2010).

[3] P. G. Baranov, A. P. Bundakova, A. A. Soltamova, S. B. Orlinskii, I. V. Borovykh, R. Zondervan, R. Verberk, and J. Schmidt, Silicon Vacancy in $\mathrm{SiC}$ as a Promising Quantum System for Single-Defect and Single-Photon Spectroscopy, Phys. Rev. B 83, 125203 (2011).

[4] D. Riedel, F. Fuchs, H. Kraus, S. Väth, A. Sperlich, V. Dyakonov, A. Soltamova, P. Baranov, V. Ilyin, and G. V. Astakhov, Resonant Addressing and Manipulation of 
Silicon Vacancy Qubits in Silicon Carbide, Phys. Rev. Lett. 109, 226402 (2012).

[5] F. Fuchs, V. A. Soltamov, S. Väth, P. G. Baranov, E. N. Mokhov, G. V. Astakhov, and V. Dyakonov, Silicon Carbide Light-Emitting Diode as a Prospective Room Temperature Source for Single Photons, Sci. Rep. 3, 1637 (2013).

[6] S. Castelletto, B. C. Johnson, and A. Boretti, Quantum Effects in Silicon Carbide Hold Promise for Novel Integrated Devices and Sensors, Adv. Opt. Mater. 1, 609 (2013).

[7] B. Somogyi and A. Gali, Computational Design of In Vivo Biomarkers, J. Phys. Condens. Matter 26, 143202 (2014).

[8] A. Muzha, F. Fuchs, N. V. Tarakina, D. Simin, M. Trupke, V. A. Soltamov, E. N. Mokhov, P. G. Baranov, V. Dyakonov, A. Krueger, and G. V. Astakhov, Room-Temperature Near-Infrared Silicon Carbide Nanocrystalline Emitters based on Optically Aligned Spin Defects, Appl. Phys. Lett. 105, 243112 (2014).

[9] G. Calusine, A. Politi, and D. D. Awschalom, Silicon Carbide Photonic Crystal Cavities with Integrated Color Centers, Appl. Phys. Lett. 105, 011123 (2014).

[10] W. F. Koehl, B. B. Buckley, F. J. Heremans, G. Calusine, and D. D. Awschalom, Room Temperature Coherent Control of Defect Spin Qubits in Silicon Carbide, Nature (London) 479, 84 (2011).

[11] V. A. Soltamov, A. A. Soltamova, P. G. Baranov, and I. I. Proskuryakov, Room Temperature Coherent Spin Alignment of Silicon Vacancies in $4 \mathrm{H}$ - and $6 \mathrm{H}$-SiC, Phys. Rev. Lett. 108, 226402 (2012).

[12] A. L. Falk, B. B. Buckley, G. Calusine, W. F. Koehl, V. V. Dobrovitski, A. Politi, C. A. Zorman, P. X. L. Feng, and D. D. Awschalom, Polytype Control of Spin Qubits in Silicon Carbide, Nat. Commun. 4, 1819 (2013).

[13] H. Kraus, V. A. Soltamov, D. Riedel, S. Väth, F. Fuchs, A. Sperlich, P. G. Baranov, V. Dyakonov, and G. V. Astakhov, Room-Temperature Quantum Microwave Emitters based on Spin Defects in Silicon Carbide, Nat. Phys. 10, 157 (2014).

[14] P. V. Klimov, A. L. Falk, B. B. Buckley, and D. D. Awschalom, Electrically Driven Spin Resonance in Silicon Carbide Color Centers, Phys. Rev. Lett. 112, 087601 (2014).

[15] A. L. Falk, P. V. Klimov, B. B. Buckley, V. Ivády, I. A. Abrikosov, G. Calusine, W. F. Koehl, A. Gali, and D. D. Awschalom, Electrically and Mechanically Tunable Electron Spins in Silicon Carbide Color Centers, Phys. Rev. Lett. 112, 187601 (2014).

[16] H. Kraus, V. A. Soltamov, F. Fuchs, D. Simin, A. Sperlich, P. G. Baranov, G. V. Astakhov, and V. Dyakonov, Magnetic Field and Temperature Sensing with Atomic-Scale Spin Defects in Silicon Carbide, Sci. Rep. 4, 5303 (2014).

[17] L.-P. Yang, C. Burk, M. Widmann, S.-Y. Lee, J. Wrachtrup, and N. Zhao, Electron Spin Decoherence in Silicon Carbide Nuclear Spin Bath, Phys. Rev. B 90, 241203 (2014).

[18] O. V. Zwier, D. O'Shea, A. R. Onur, and C. H. van der Wal, All-Optical Coherent Population Trapping with Defect Spin Ensembles in Silicon Carbide, Sci. Rep. 5, 10931 (2015).

[19] A. L. Falk, P. V. Klimov, V. Ivády, K. Szász, D. J. Christle, W.F. Koehl, A. Gali, and D. D. Awschalom, Optical Polarization of Nuclear Spins in Silicon Carbide, Phys. Rev. Lett. 114, 247603 (2015).
[20] S. G. Carter, Ö. O. Soykal, P. Dev, S. E. Economou, and E. R. Glaser, Spin Coherence and Echo Modulation of the Silicon Vacancy in 4H-SiC at Room Temperature, Phys. Rev. B 92, 161202 (2015).

[21] D. Simin, F. Fuchs, H. Kraus, A. Sperlich, P. G. Baranov, G. V. Astakhov, and V. Dyakonov, High-Precision AngleResolved Magnetometry with Uniaxial Quantum Centers in Silicon Carbide, Phys. Rev. Applied 4, 014009 (2015).

[22] S.-Y. Lee, M. Niethammer, and J. Wrachtrup, Vector Magnetometry based on $S=3 / 2$ Electronic Spins, Phys. Rev. B 92, 115201 (2015).

[23] S. Castelletto, B. C. Johnson, V. Ivády, N. Stavrias, T. Umeda, A. Gali, and T. Ohshima, A Silicon Carbide Room-Temperature Single-Photon Source, Nat. Mater. 13, 151 (2013).

[24] S. Castelletto, B. C. Johnson, C. Zachreson, D. Beke, I. Balogh, T. Ohshima, I. Aharonovich, and A. Gali, Room Temperature Quantum Emission from Cubic Silicon Carbide Nanoparticles, ACS Nano 8, 7938 (2014).

[25] D. J. Christle, A. L. Falk, P. Andrich, P. V. Klimov, J. ul Hassan, N. T. Son, E. Janzén, T. Ohshima, and D. D. Awschalom, Isolated Electron Spins in Silicon Carbide with Millisecond Coherence Times, Nat. Mater. 14, 160 (2015).

[26] M. Widmann, S.-Y. Lee, T. Rendler, N. T. Son, H. Fedder, S. Paik, L.-P. Yang, N. Zhao, S. Yang, I. Booker, A. Denisenko, M. Jamali, S. Ali Momenzadeh, I. Gerhardt, T. Ohshima, A. Gali, E. Janzén, and J. Wrachtrup, Coherent Control of Single Spins in Silicon Carbide at Room Temperature, Nat. Mater. 14, 164 (2015).

[27] F. Fuchs, B. Stender, M. Trupke, D. Simin, J. Pflaum, V. Dyakonov, and G. V. Astakhov, Engineering Near-Infrared Single-Photon Emitters with Optically Active Spins in Ultrapure Silicon Carbide, Nat. Commun. 6, 7578 (2015).

[28] A. Lohrmann, N. Iwamoto, Z. Bodrog, S. Castelletto, T. Ohshima, T. J. Karle, A. Gali, S. Prawer, J. C. McCallum, and B. C. Johnson, Single-Photon Emitting Diode in Silicon Carbide, Nat. Commun. 6, 7783 (2015).

[29] J. R. Maze, P. L. Stanwix, J. S. Hodges, S. Hong, J. M. Taylor, P. Cappellaro, L. Jiang, M. V. Gurudev Dutt, E. Togan, A. S. Zibrov, A. Yacoby, R. L. Walsworth, and M. D. Lukin, Nanoscale Magnetic Sensing with an Individual Electronic Spin in Diamond, Nature (London) 455, 644 (2008).

[30] G. Balasubramanian, I. Y. Chan, R. Kolesov, M. Al-Hmoud, J. Tisler, C. Shin, C. Kim, A. Wojcik, P. R. Hemmer, A. Krueger, T. Hanke, A. Leitenstorfer, R. Bratschitsch, F. Jelezko, and J. Wrachtrup, Nanoscale Imaging Magnetometry with Diamond Spins under Ambient Conditions, Nature (London) 455, 648 (2008).

[31] T. Wolf, P. Neumann, K. Nakamura, H. Sumiya, T. Ohshima, J. Isoya, and J. Wrachtrup, Subpicotesla Diamond Magnetometry, Phys. Rev. X 5, 041001 (2015).

[32] N. Mizuochi, S. Yamasaki, H. Takizawa, N. Morishita, T. Ohshima, H. Itoh, and J. Isoya, Continuous-Wave and Pulsed EPR Study of the Negatively Charged Silicon Vacancy with $S=3 / 2$ and $\mathrm{C}_{3 v}$ Symmetry in n-Type $4 H$ SiC, Phys. Rev. B 66, 235202 (2002).

[33] B. P. Lanyon, M. Barbieri, M. P. Almeida, T. Jennewein, T. C. Ralph, K. J. Resch, G. J. Pryde, J. L. O’Brien, A. Gilchrist, and A. G. White, Simplifying Quantum Logic 
Using Higher-Dimensional Hilbert Spaces, Nat. Phys. 5, 134 (2009).

[34] H. Clevenson, M. E. Trusheim, C. Teale, T. Schröder, D. Braje, and D. Englund, Broadband Magnetometry and Temperature Sensing with a Light-Trapping Diamond Waveguide, Nat. Phys. 11, 393 (2015).

[35] V. Shah, S. Knappe, P. D. D. Schwindt, and J. Kitching, Subpicotesla Atomic Magnetometry with a Microfabricated Vapour Cell, Nat. Photonics 1, 649 (2007).

[36] E. Sörman, N. Son, W. Chen, O. Kordina, C. Hallin, and E. Janzén, Silicon Vacancy Related Defect in $4 \mathrm{H}$ and $6 \mathrm{H} \mathrm{SiC,}$ Phys. Rev. B 61, 2613 (2000).

[37] S. Y. Karpov, A. V. Kulik, I. A. Zhmakin, Y. N. Makarov, E. N. Mokhov, M. G. Ramm, M. S. Ramm, A. D. Roenkov, and Y. A. Vodakov, Analysis of Sublimation Growth of Bulk SiC Crystals in Tantalum Container, J. Cryst. Growth 211 , 347 (2000).

[38] T. C. Hain, F. Fuchs, V. A. Soltamov, P. G. Baranov, G. V. Astakhov, T. Hertel, and V. Dyakonov, Excitation and Recombination Dynamics of Vacancy-Related Spin Centers in Silicon Carbide, J. Appl. Phys. 115, 133508 (2014).

[39] H. J. von Bardeleben, J. L. Cantin, L. Henry, and M. F. Barthe, Vacancy Defects in p-Type 6H-SiC Created by Low-Energy Electron Irradiation, Phys. Rev. B 62, 10841 (2000).

[40] E. van Oort and M. Glasbeek, Fluorescence Detected LevelAnticrossing and Spin Coherence of a Localized Triplet State in Diamond, Chem. Phys. 152, 365 (1991).

[41] J. P. D. Martin, N. B. Manson, D. C. Doetschman, M. J. Sellars, R. Neuhaus, and E. Wilson, Spectral Hole Burning and Raman Heterodyne Signals Associated with an Avoided Crossing in the NV Centre in Diamond, J. Lumin. 86, 355 (2000).

[42] P. G. Baranov and N. G. Romanov, Magnetic Resonance in Micro- and Nanostructures, Appl. Magn. Reson. 21, 165 (2001).

[43] R. J. Epstein, F. M. Mendoza, Y. K. Kato, and D. D. Awschalom, Anisotropic Interactions of a Single Spin and Dark-Spin Spectroscopy in Diamond, Nat. Phys. 1, 94 (2005).
[44] L. J. Rogers, R. L. McMurtrie, M. J. Sellars, and N. B. Manson, Time-Averaging within the Excited State of the Nitrogen-Vacancy Centre in Diamond, New J. Phys. 11, 063007 (2009).

[45] J. P. Tetienne, L. Rondin, P. Spinicelli, M. Chipaux, T. Debuisschert, J.F. Roch, and V. Jacques, MagneticField-Dependent Photodynamics of Single NV Defects in Diamond: An Application to Qualitative All-Optical Magnetic Imaging, New J. Phys. 14, 103033 (2012).

[46] V. A. Soltamov, B. V. Yavkin, D. O. Tolmachev, R. A. Babunts, A. G. Badalyan, V. Y. Davydov, E. N. Mokhov, I. I. Proskuryakov, S. B. Orlinskii, and P. G. Baranov, Optically Addressable Silicon Vacancy-Related Spin Centers in Rhombic Silicon Carbide with High Breakdown Characteristics and ENDOR Evidence of Their Structure, Phys. Rev. Lett. 115, 247602 (2015).

[47] N. Mizuochi, S. Yamasaki, H. Takizawa, N. Morishita, T. Ohshima, H. Itoh, and J. Isoya, EPR Studies of the Isolated Negatively Charged Silicon Vacancies in n-Type $4 H$ - and 6H-SiC: Identification of $\mathrm{C}_{3 v}$ Symmetry and Silicon Sites, Phys. Rev. B 68, 165206 (2003).

[48] G. L. Bir and G. E. Pikus, Symmetry and Strain-Induced Effects in Semiconductors (Wiley, New York, 1974).

[49] N. S. Averkiev, V. M. Asnin, Y. N. Lomasov, G. E. Pikus, A. A. Rogachev, and N. A. Rud, Radiation Polarization of a Coupled Exciton in Ge(As) in a Longitudinal Magnetic Field, Sov. Phys. Solid State 23, 1851 (1981).

[50] M. V. Durnev, M. M. Glazov, E. L. Ivchenko, M. Jo, T. Mano, T. Kuroda, K. Sakoda, S. Kunz, G. Sallen, L. Bouet, X. Marie, D. Lagarde, T. Amand, and B. Urbaszek, Magnetic Field Induced Valence Band Mixing in [111] Grown Semiconductor Quantum Dots, Phys. Rev. B 87, 085315 (2013).

[51] R. Schirhagl, K. Chang, M. Loretz, and C. L. Degen, Nitrogen-Vacancy Centers in Diamond: Nanoscale Sensors for Physics and Biology, Annu. Rev. Phys. Chem. 65, 83 (2014).

[52] E. L. Ivchenko and G. E. Pikus, Superlattices and Other Heterostructures: Symmetry and Optical Phenomena, 2nd ed. (Springer-Verlag, Berlin, 1997). 